\title{
A GLOBAL RELATION OF FIRE SMOKE RE-CIRCULATION BEHAVIOUR IN URBAN STREET CANYONS
}

\author{
Longhua $\mathrm{HU}^{\mathrm{a}}$, Xiaochun $Z \mathrm{HANG}^{\mathrm{a}}$, Wei ZHU ${ }^{\mathrm{b}}$, Zhi NING ${ }^{\mathrm{c}}$, Fei TANG \\ ${ }^{a}$ State Key Laboratory of Fire Science, University of Science and Technology of China, Hefei, China \\ ${ }^{b}$ Beijing Research Center of Urban System Engineering, Beijing, China \\ cSchool of Energy and Environment, City University of Hong Kong, Hong Kong, China
}

Received 10 Aug 2012; accepted 07 Nov 2012

\begin{abstract}
Street canyon, formed by buildings along two sides of a road, is an important and ubiquitous architectural element in the metropolises. When a fire occurs in an urban street canyon, a critical serious phenomenon is found to happen where the uprising fire smoke is re-circulated back into the base of the street canyon by the wind flow beyond a critical velocity. Theoretical analysis is derived based on Froude number (Fr), by balancing the inertial force of the wind flow to the buoyancy strength of the fire smoke. It is found that the critical re-circulation wind velocity is proportional to an integrated global parameter $\dot{Q}^{1 / 3} H^{-1 / 3}$. Large Eddy Simulations (LES) are carried out by Fire Dynamics Simulator (FDS). The critical Froude number (Fr) is found to be about $0.7 \sim 0.8$. The simulation results are well collapsed by the generalized theoretical relation. It is further revealed that the non-dimensional critical re-circulation wind velocity $\frac{u_{c}}{\dot{Q}^{1 / 3} H^{-1 / 3}}\left(\frac{\rho c_{p} T_{\infty}}{g}\right)^{1 / 3}$. against aspect ratio of street canyons in the skimming flow pattern $(\mathrm{W} / \mathrm{H}<1.43)$ falls into two behavioural regimes, where it firstly increases then remains constant with the increase in street canyon aspect ratio $(\mathrm{W} / \mathrm{H})$ with a turning point at $\mathrm{W} / \mathrm{H}=1$. A global non-dimensional relation is finally achieved for the critical re-circulation wind velocity $(u)$, fire heat release rate $(Q)$ and its height $(H)$ to the top of the street canyon as well as the street canyon aspect ratio $(\mathrm{W} / \mathrm{H})$.
\end{abstract}

Keywords: fire smoke dispersion, street canyon, critical re-circulation wind velocity, Froude number.

\section{Introduction}

Fire safety is one of the important considerations of civil engineering and it has been widely studied by the researchers (Jonaitis, Papinigis 2005; Konecki, Półka 2009). Simultaneously, smoke movement during a building fire attracts more and more attention in recent years (Delichatsios 2004; Chow, Li 2010; Sun et al. 2011). Street canyon (Vardoulakisr et al. 2003) is one of the basic architectural elements in metropolises, which is formed by large number of high buildings built along two sides of a road.

Hazardous pollutant dispersion and the air quality concerning environmental health in street canyons, as related closely to human activity, have attracted extensive attention in recently years (Kim, Baik 2001, 2010; Alarie 2002). Both structure of street canyon and wind play important roles in such hazardous dispersion (Lee, Park 1994; Xia, Leung 2001; Liu et al. 2004; Chang 2006; Tominaga et al. 2008). It is known that the pollutants emitted at the base of a street canyon will be recirculated inside the street canyon and accumulated to high levels when the street canyon is narrow, for example, in the case its aspect ratio $(\mathrm{W} / \mathrm{H})$ is less than 1.43 with so called "skimming flow pattern" (Liu, Barth 2002; Liu et al. 2003; Baker et al. 2004). In recent years, buoyancy effect on such pollutant dispersion in street canyons has attracted increasing attention (Sini et al. 1996; Uehara et al. 2000; Markatos et al. 2009), for its complex interaction with wind. For example, Sini et al. (1996) investigated the effects of the wall heat flux due to the solar heating of ground or building walls on pollutant dispersion within a street canyon. Kim and Baik investigated the flow characteristics and its effect on pollutant dispersion with bottom heating (Kim, Baik 2001) and with both bottom and roof heating (Kim, Baik 2010) within a street canyon.

However, these reported works only considered a very weak natural buoyancy condition induced by solar heating. More recently, a more catastrophic scenario (Besserre, Delort 1997; Alarie 2002) has been investigated under which the hazardous pollutant is produced by a catastrophic fire at the base of a street canyon (Hu et al. 2009) or in an adjacent building (Hu et al.

Corresponding author: Longhua $\mathrm{Hu}$

E-mail: hlh@ustc.edu.cn 
2010) along a street canyon. The fire-yield hazardous pollutants include soot (in solid phase), which degrades visibility and causes inflammation of the respiratory system, as well as $\mathrm{CO}_{x}, \mathrm{SO}_{x}, \mathrm{NO}_{x}$ (in gaseous phase), which are poisonous to people. These will pose serious harm to human health. Recent studies (Besserre, Delort 1997) have proved that the main cause of death during urban fires is poisoning by smoke.

Complex interaction of the external wind flow with fire-induced buoyant flow should be accounted for, which is much more complex than that produced naturally by solar effect, in characterizing the dispersion of fire smoke in urban atmosphere. For the natural buoyancy effect induced by solar energy, the heating of the street canyon base is uniform, resulting in a relative spatially uniform temperature rise usually less than $50{ }^{\circ} \mathrm{C}$ along the whole ground base surface. However, a fire source is separately self-standing with (flame) temperature rise over $500{ }^{\circ} \mathrm{C}$ contributing a much stronger buoyancy. A fire source emits pollutant smoke and, at the same time, provides inherently strong buoyancy to influence their transportation characteristics remarkably. This has not been investigated much in the past.

Interesting and important characteristics have been found recently (Hu et al. 2009, 2010) for such fire scenarios as under wind over a critical velocity, i.e. hazardous pollutant smoke will be re-circulated back to the street canyon as shown in Figure 1, which will be toxic so harmful to the pedestrians inside a street canyon and may also cause traffic accidents more easily due to reduced visibility by the smoke soot. However, such pollutant smoke will be initially vented out of the street canyon by the aid of its own buoyancy if the buoyancy strength of the fire source is relatively strong or the wind velocity is relative weak. In the first step of our work (Hu et al. 2009), the scenario has been studied for the fire source being at the street canyon ground base with different buoyant strength, and it was found that the critical re-circulation wind velocity increases with the HRR of the fire source. In the following work (Hu et al. 2010), the scenario of room fire in the adjacent building emitting pollutant smoke into the street canyon has been studied. It is found that the critical re-circulation wind velocity is proportional to $-1 / 3$ power of the height from the fire floor to the top of the street canyon. In those former investigations, a street canyon with a fixed dimension of $18 \mathrm{~m}$ wide and $18 \mathrm{~m}$ high was considered. Details on temperature, soot and $\mathrm{CO}$ concentration field related to the transportation of the fire-induced pollutant smoke were presented in these former investigations (Hu et al. 2009, 2010).

However, these two former works considered the parameters separately for independently different fire source scenarios, respectively. And the effect of aspect ratio $(\mathrm{W} / \mathrm{H})$ of the street canyon was not considered. The current work is a continued effort aiming to generalize and complement those studies by the following attempts:

1) Theoretical analysis is performed to account for the interaction of the wind flow effect with the fire smoke buoyancy and their balance (represented by non-dimensional physical parameter, Froude number) to interpret and characterize the above recirculation phenomenon and characteristics. This is to find a generalized relation of critical re-circulation wind velocity as a universal parameter to integrate different fire source scenarios and incorporate independent parameters i.e. HRR of fire source $(\dot{Q})$ and height $(H)$ to the top of street canyon) for a generalized application.

(2) CFD LES simulation is carried out to find out the critical re-circulation wind velocity, and thus the generalized critical Froude number, for various scenarios of fire sources and street canyons dimensions.

(3) The CFD simulation results for the above scenario along with those achieved in the former investigations are consolidated together to compare with and validate the theoretically deduced relation in its generalization. The variation of non-dimensional critical wind velocity against aspect ratio $(\mathrm{W} / \mathrm{H})$ of the street canyon is also identified.

\section{Theoretical consideration}

For pollutant fire smoke dispersion in an urban atmosphere, wind flow plays an important role, due to its complex interaction with the urban structure and buoyancy of the fire. This can be generalized by a local Froude number (Fr) (Hu et al. 2010):

$$
F r=\frac{u}{\sqrt{\left(\Delta T_{z} / T_{\infty}\right) g z}},
$$

where: $u$ is the wind velocity; $\Delta T$ is the local temperature rise above the ambient temperature $\rho_{\infty} ; g$ is the acceleration due to gravity; and $z$ is the characteristic height. Under the critical condition of re-circulation, the competition of fire smoke buoyancy with inertial strength of the wind flow should achieve a balance and the Froude number should reach a certain constant critical value. This indicates:

$$
u_{c} \infty \sqrt{\Delta T_{H} H},
$$

where: $H$ is the height of street canyon if the fire source is positioned at street ground base (Fig. 1b), or its height to the top of the street canyon if it is in the windward adjacent building (Fig.1a, left building). It means that the critical re-circulation wind velocity is in relation to both the height of urban street canyon and the fire smoke temperature at the top of urban street canyon. However, the temperature rise should relate to HRR $(\dot{Q})$ of the source and height $z$, which can be further calculated by the following self-preserving equations based on conservation of the energy, mass and momentum of the fire smoke plume.

Based on the observation in Figure $1 \mathrm{~b}$ that the buoyant plume remains straight vertical, as similar to 


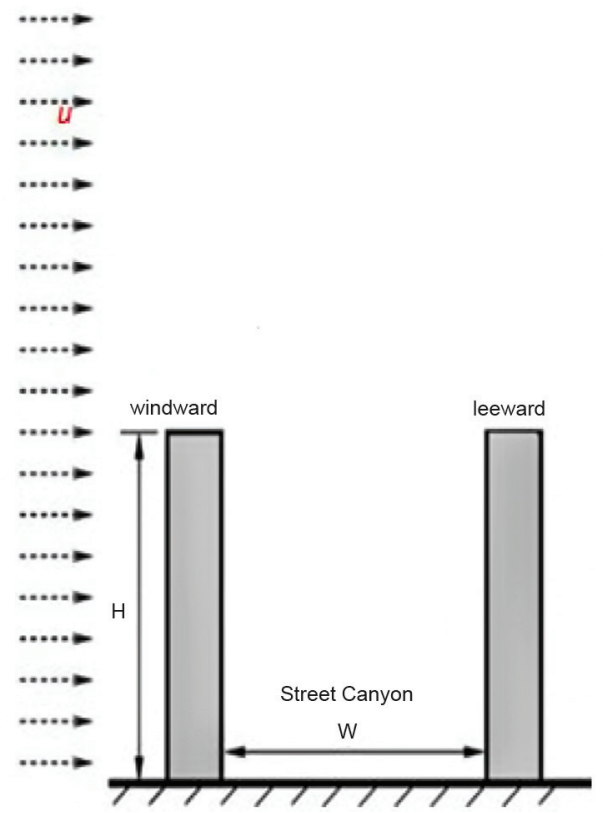

(a) an idealized street canyon model

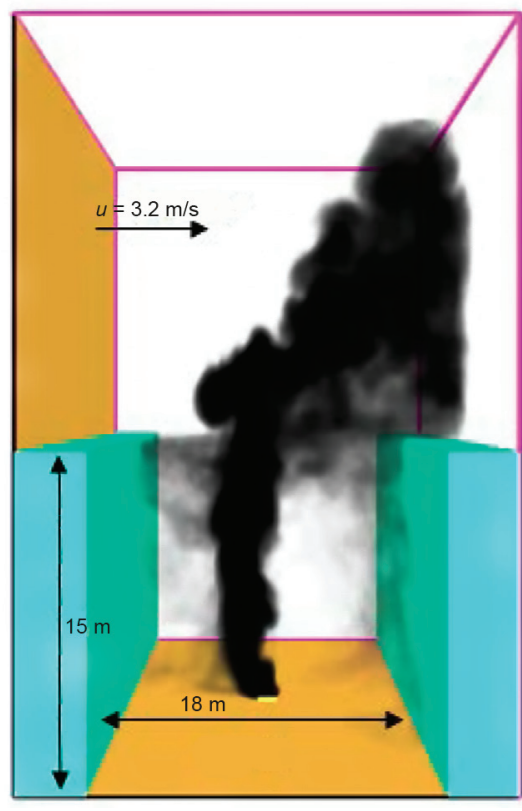

(b) re-circulation (soot field)
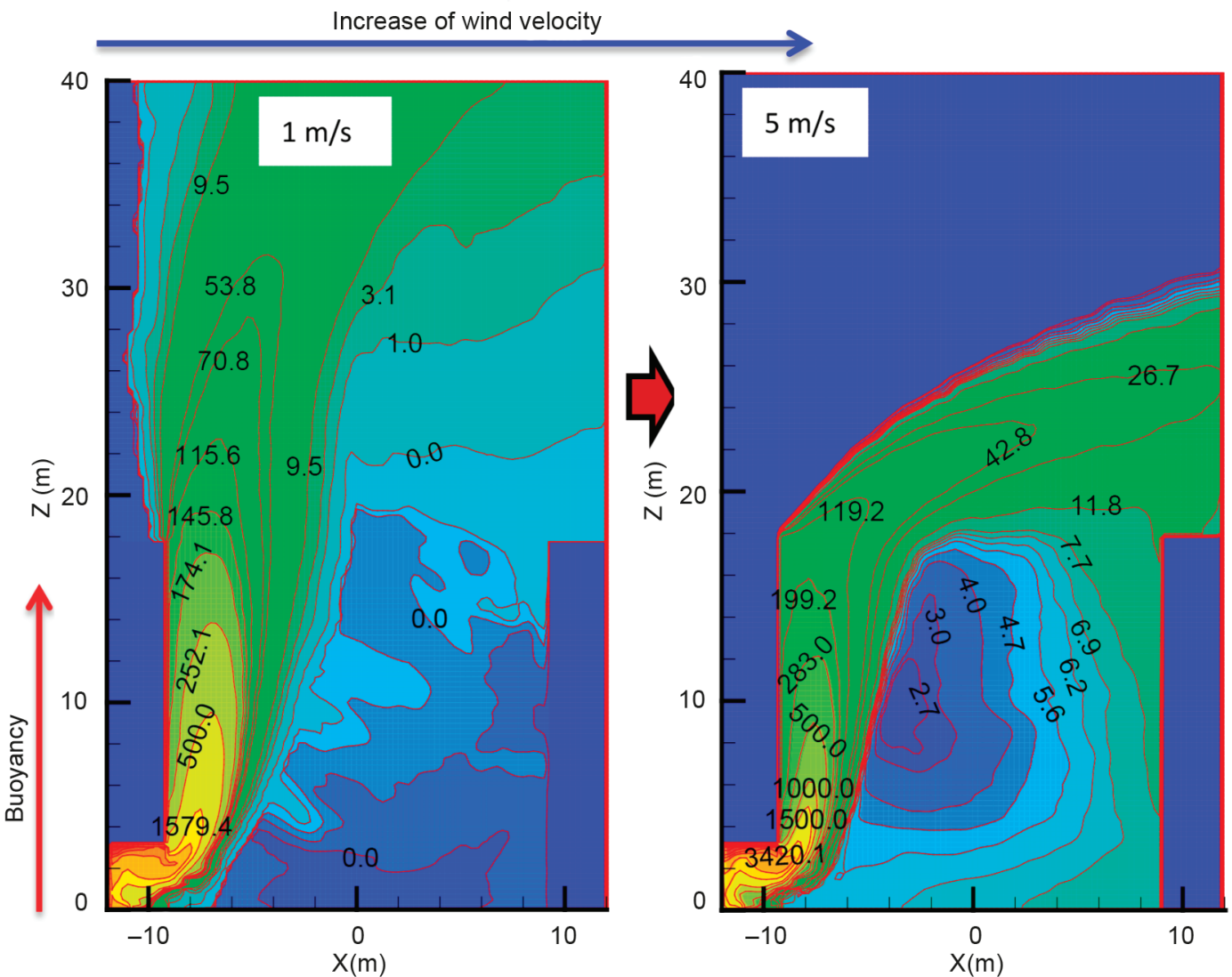

(c) re-circulation ( $\mathrm{CO}$ concentration contour, ppm)

Fig. 1. Critical re-circulation behaviour for fire-induced pollutant smoke (soot, $\mathrm{CO}$ ) dispersion in atmosphere of an urban street canyon 
that in no wind, it is assumed that the conservation laws of a buoyant plume in no wind can still characterize its properties. The plume parameters changes vertically due to the buoyancy-induced entrainment of fresh air into the plume.

For the conservation of energy of the fire plume, the convection of the buoyancy flux at any height $z$ should maintain constant equal to initial buoyancy strength (quantified by the Heat Release Rate, $\dot{Q}$ ) of the fire source (the only possible heat loss is due to radiation loss, which is usually taken as negligible in the fire plume dynamics theory):

$$
\dot{Q}=c_{p} \dot{m} \Delta T_{z}=c_{p} \rho_{z} w \pi r_{z}^{2} \Delta T_{z}
$$

where: $r_{z}$ is the plume radius; $w$ is the uprising velocity of the plume at height $z$. In fact, for such pollutant dispersion, the dominant mechanism of spatial change of the temperature is physically controlled by the entraining transportation of fresh ambient air into the pollutant flow. The plume temperature and uprising velocity should both decrease with height due to the buoyancy-induced turbulent entrainment of fresh air into the pollutant plume at its edge, resulting an increase of mass flow rate $m$ with height and therefore a lowering down of the buoyancy. So, for the conservation of mass:

$$
\frac{d(\dot{m})}{d z}=\frac{d\left(\rho w \pi r_{z}^{2}\right)}{d z}=\rho_{\infty} \alpha w \cdot 2 \pi r_{z}
$$

where $\alpha$ is the air entrainment coefficient.

Based on Boussinesq assumption $\left(\rho_{\mathrm{z}} \approx \rho_{\infty}\right)$ :

$$
\frac{d\left(w r_{z}^{2}\right)}{d z}=2 \alpha w r_{z}
$$

For the conservation of momentum:

$$
\frac{d\left(w^{2} r_{z}^{2}\right)}{d z}=\frac{\Delta \rho}{\rho} g r_{z}^{2}=\frac{\Delta T}{T_{\infty}} g r_{z}^{2} .
$$

Substituting Eqn (3) into (6), it gives:

$$
\frac{d\left(w^{2} r_{z}^{2}\right)}{d z}=\frac{\dot{Q} g}{\rho_{\infty} c_{p} T_{\infty} w \pi} \text {. }
$$

Solving Eqns (4) and (7) based on the initial conditions of $z=0, r_{z}=0$ and $w=0$, it can be achieved:

$$
w \propto \dot{Q}^{1 / 3} z^{-1 / 3}, r_{z} \propto z .
$$

Substituting Eqn (8) into Eqn (3), it gives the well-known relation of plume temperature with buoyant strength of the fire and height:

$$
\Delta T_{z} \propto \dot{Q}^{2 / 3} z^{-5 / 3} .
$$

In fact, similar power law relations can be also achieved for the scenario of buoyant plume spills from the adjacent room fire and develops along the building facade (Tang et al. 2011). Further substituting Eqn (9) into Eqn (2), it can be achieved that at height of $H$ from fire source to the top of the street canyon, the critical re-circulation wind velocity has:

$$
u_{c} \propto \dot{Q}^{1 / 3} \cdot H^{-1 / 3} .
$$

This is a generalised relation applicable for any scenario of buoyancy strength $(Q)$ of the fire and its height to the top of the street canyon $(H)$. In our former investigation of fire occurred in the adjacent building (Hu et al. 2010), it has been proved that $u_{c} \infty H^{-1 / 3}$. In the subsequent sections, the following points will be further checked for validation of Eqn (10) by CFD LES simulations as well as to find out a general global relation including the aspect ratio $(\mathrm{W} / \mathrm{H})$ of the street canyon:

- For fire source positioned at the street ground base, whether the variation of the critical re-circulation wind velocity still follows $u_{c} \infty H^{-1 / 3}$ when changing the height of street canyon?

- Whether the critical re-circulation wind velocities for all scenarios including fire source at the street ground base with different buoyancy strength $(Q)$ and height of the street canyon $(H)$, and fire source at the different floor of adjacent building with different height to the top of the street canyon, can be all collapsed by the integrated parameter of $\dot{Q}^{1 / 3} \cdot H^{-1 / 3}$ with a linear proportional relationship indicating a successful generalized application?

- How the critical re-circulation wind velocity varies with the street canyon $(\mathrm{W} / \mathrm{H})$ of the street canyon? This is to be combined with above findings as to find out a global relation for the critical re-circulation wind velocity $(u)$, fire heat release rate $(\dot{Q})$ and its height $(H)$ to the top of the street canyon as well as the street canyon aspect ratio $(\mathrm{W} / \mathrm{H})$.

\section{Numerical method and model setup}

\subsection{Numerical methodology}

Turbulence methods used in CFD generally include Reynolds Averaging Navier-Stokes equation (RANS) method, Large Eddy Simulation (LES) and Direct Numerical Simulation (DNS) (Besserre, Delort 1997; Alarie 2002; $\mathrm{Hu}$ et al. 2009). Large Eddy Simulation (LES) is able to predict the unsteadiness and intermittency which is the most important feature of a strong turbulent buoyancydriven flow. It has been recently widely applied to simulate the turbulent pollutant transport in street canyons and fire-induced flow in fire scenarios. In LES, the large eddy turbulence is directly computed while the small turbulent motions are modelled by Sub-Grid Models (SGM).Walton et al. (Walton, Cheng 2002; Walton et al. 2002) demonstrated that the LES predictions could exhibit agreement with the experimental results very well in the street canyon. 
Fire Dynamics Simulator (FDS) (McGrattan et al. 2009, 2010) which is developed by National Institute of Standards and Technology (NIST), solves numerically a form of the Navier-Stokes equations for thermally driven flow. It is now a popular CFD tool in fire related researches, as well as used to simulate the pollutant concentration and flow field in urban street canyons (Chang 2006; Hu et al. $2009,2010)$. The governing equations which consist of conservation of mass, momentum and the transport of sensible enthalpy for LES simulation can be found in (McGrattan et al. 2009, 2010).

Conservation of mass and transport for individual gaseous species, $Y_{\alpha}$ :

$$
\frac{\partial}{\partial t}\left(\rho Y_{\alpha}\right)+\nabla \cdot \rho Y_{\alpha} \vec{u}=\nabla \cdot \rho D_{\alpha} \nabla Y_{\alpha}+\dot{m}_{\alpha}^{\prime \prime \prime}+\dot{m}_{b, \alpha}^{\prime \prime \prime}
$$

where: $Y_{\alpha}$ is the mass fraction of the individual gaseous species; $\dot{m}_{b, \alpha}^{\prime \prime \prime}$ is the production rate of species $\alpha$ by evaporating droplets/particles; and $\dot{m}_{\alpha}^{\prime \prime \prime}$ is the mass production rate of species $\alpha$ per unit volume.

Conservation of momentum:

$$
\frac{\partial}{\partial t}(\rho \vec{u})+\nabla \cdot \rho \vec{u} \vec{u}+\nabla p=\rho g+\vec{f}_{b}+\nabla \cdot \tau_{i j} .
$$

Transport of sensible enthalpy, $h_{s}$ :

$$
\frac{\partial}{\partial t}\left(\rho h_{s}\right)+\nabla \cdot \rho h_{s} \vec{u}=\frac{D p}{D t}+\dot{q}^{\prime \prime \prime}-\dot{q}_{b}^{\prime \prime \prime}-\nabla \cdot \dot{\vec{q}}^{\prime \prime}+\varepsilon
$$

The convergence criteria in FDS model is not based on residue judgement, as used in many other CFD models, especially those based on RANS turbulence model. In FDS, the Courant-Friedrichs-Lewy (CFL) criterion is used along with a self-varying time step (McGrattan et al. 2009) for justifying the computational convergence. The physical meaning of such a convergence criteria is that when computing a wave crossing a discrete grid, the time step must be less than the time for the wave to travel adjacent grid points. This criterion is more important for large-scale calculations where convective transport dominates the diffusive one. In FDS, the estimated velocities are tested at each time step to ensure that the following CFL criterion is satisfied (McGrattan et al. 2010):

$$
\delta t \cdot \max \left(\frac{\left|u_{i j k}\right|}{\delta x}, \frac{\left|v_{i j k}\right|}{\delta y}, \frac{\left|w_{i j k}\right|}{\delta z}\right)<1 .
$$

During the calculation, the time step is varying and constrained by the convective and diffusive transport speed to ensure that the CFL condition is satisfied at each time step (McGrattan et al. 2009). The initial time step is set automatically in FDS by the size of a grid cell divided by the characteristic velocity of the flow. The default value of the initial time step is $5(\delta x \delta y \delta z)^{1 / 3} / \sqrt{g H}$ (McGrattan et al. 2009), where $x, y$, and $z$ are the dimensions of the smallest grid cell, $H$ is the height of the computational domain, and $g$ is the acceleration due to gravity. The time step will eventually get to be a quasi-steady value when the flow field reaches a quasi-steady state.

\subsection{Physical model setup}

LES simulations are performed by FDS for street canyons in different heights of $9 \mathrm{~m}, 12 \mathrm{~m}, 15 \mathrm{~m}, 18 \mathrm{~m}, 21 \mathrm{~m}$ and $24 \mathrm{~m}$, keeping the aspect ratio $(\mathrm{W} / \mathrm{H})$ of the street canyon to be 1 , or in different width of $9 \mathrm{~m}, 12 \mathrm{~m}, 15 \mathrm{~m}, 18 \mathrm{~m}$, $21 \mathrm{~m}, 24 \mathrm{~m}$ and $27 \mathrm{~m}$ for the same height of $18 \mathrm{~m}$ with aspect ratios $(\mathrm{W} / \mathrm{H})$ in range of 0.5 to 1.5 , to achieve the wind-driven "skimming flow pattern" $(\mathrm{W} / \mathrm{H}<1.43)$ in the street canyon (Nakamura, Oke 1988). The flow behaviour pattern inside the street canyon had been identified (Nakamura, Oke 1988) based on the aspect ratios. The turning point between the skimming flow pattern which is characterized by a stable and isolated recirculation large vortex at the street canyon centre, and the wake interference flow pattern where the wake behind the windward building is disturbed by the large vortex formed in front of the windward building, was $\mathrm{H} / \mathrm{W}=0.7$ i.e. $\mathrm{W} / \mathrm{H} \approx 1.43$. The skimming flow pattern is widely focused in the former researches due to its special character of a large vortex formed inside the street canyon and the importance of this behaviour in pollutant dispersion. An inlet velocity boundary condition is set at the left side of the simulation domain. The simulation conditions are summarized in Table 1. The top and the other three sides of the domain are all set to be naturally opened with no initial velocity boundary condition specified. The HRR $(\dot{Q})$ of the fire source is $5 \mathrm{MW}$, a typical burning car's HRR. The grid size is uniformly $0.25 \mathrm{~m}$ in the three spatial directions as shown in Figure 2 and this grid size setting satisfied the aforementioned criterion during all of the simulated cases.

The boundary conditions and the grid system are set to be the same as the former works (Hu et al. 2009, 2010), in which the validation of the CFD model, including the turbulence statistics of the flow characteristics, and the simulation results on the field distribution of smoke temperature, soot and $\mathrm{CO}$ concentration, have been reported in details (Hu et al. 2009, 2010). A typical timeevolution of the time step and the corresponding maximum CFL number for the LES simulation is shown in Figure 3. It is observed that the CFL numbers during the simulation are less than 1 . The CFL criterion is successfully satisfied.

\section{Results and discussion}

\subsection{Smoke field and $\mathrm{CO}$ concentration contours}

Four distinct regimes were identified in the former work (Hu et al. 2009) to categorize the fire smoke dispersion pattern characteristics under different levels of wind velocities. The simulation results of soot field are shown in Figure 4 and the $\mathrm{CO}$ concentration contours in Figure 5 under different wind velocities. The smoke plume tilts toward windward building along with the increase in the wind velocity. As shown in these figures, the smoke 
Table 1. Summary of simulation cases

\begin{tabular}{|c|c|c|c|c|c|c|c|}
\hline \multicolumn{3}{|c|}{$\begin{array}{l}\text { Street canyon dimensions } \\
(\mathrm{m})\end{array}$} & \multicolumn{5}{|c|}{ Wind velocity $(\mathrm{m} / \mathrm{s})$} \\
\hline W & $\mathrm{H}$ & $\begin{array}{l}\text { Aspect ratio } \\
(\mathrm{W} / \mathrm{H})\end{array}$ & & & & & \\
\hline 9 & 18 & 0.5 & 2.2 & 2.3 & 2.4 & 2.5 & 2.6 \\
\hline 12 & 18 & $2 / 3$ & 2.4 & 2.5 & 2.6 & 2.7 & 2.8 \\
\hline 15 & 18 & $5 / 6$ & 2.6 & 2.7 & 2.8 & 2.9 & 3.0 \\
\hline 18 & 18 & 1 & 2.8 & 2.9 & 3.0 & 3.1 & 3.2 \\
\hline 21 & 18 & $7 / 6$ & 2.9 & 3.0 & 3.1 & 3.2 & 1 \\
\hline 24 & 18 & $4 / 3$ & 2.9 & 3.0 & 3.1 & 3.2 & 1 \\
\hline 27 & 18 & 1.5 & 2.9 & 3.0 & 3.1 & 3.2 & 1 \\
\hline 9 & 9 & & 3.6 & 3.7 & 3.8 & 3.9 & 1 \\
\hline 12 & 12 & & 3.4 & 3.5 & 3.6 & 3.7 & 1 \\
\hline 15 & 15 & & 3.1 & 3.2 & 3.3 & 3.4 & 1 \\
\hline 18 & 18 & 1 & 2.9 & 3.0 & 3.1 & 3.2 & 1 \\
\hline 21 & 21 & & 2.7 & 2.8 & 2.9 & 3.0 & 1 \\
\hline 24 & 24 & & 2.6 & 2.7 & 2.8 & 2.9 & 1 \\
\hline
\end{tabular}

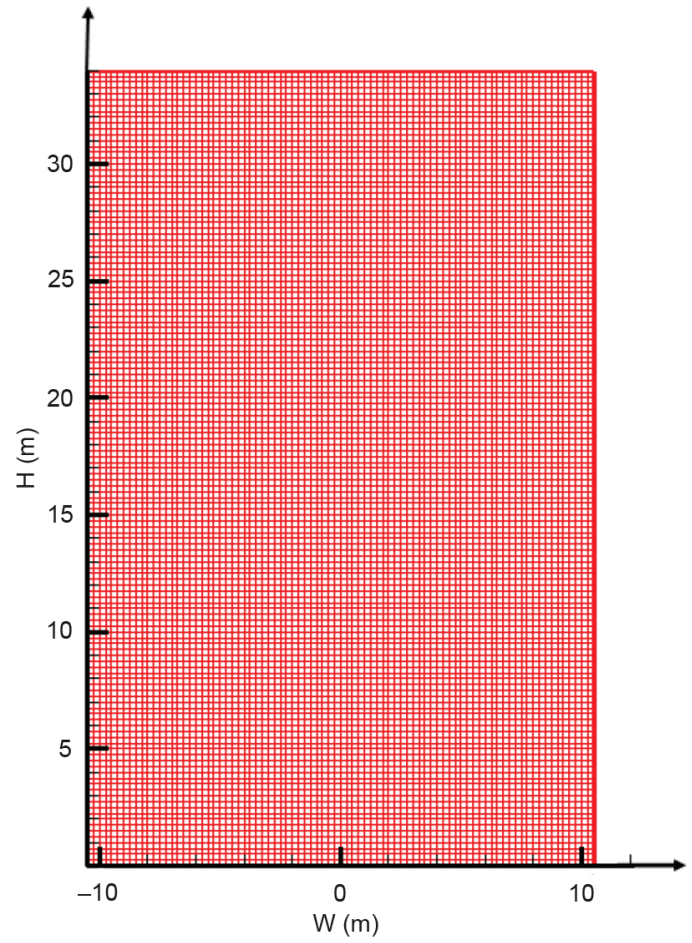

Fig. 2. The grid size system

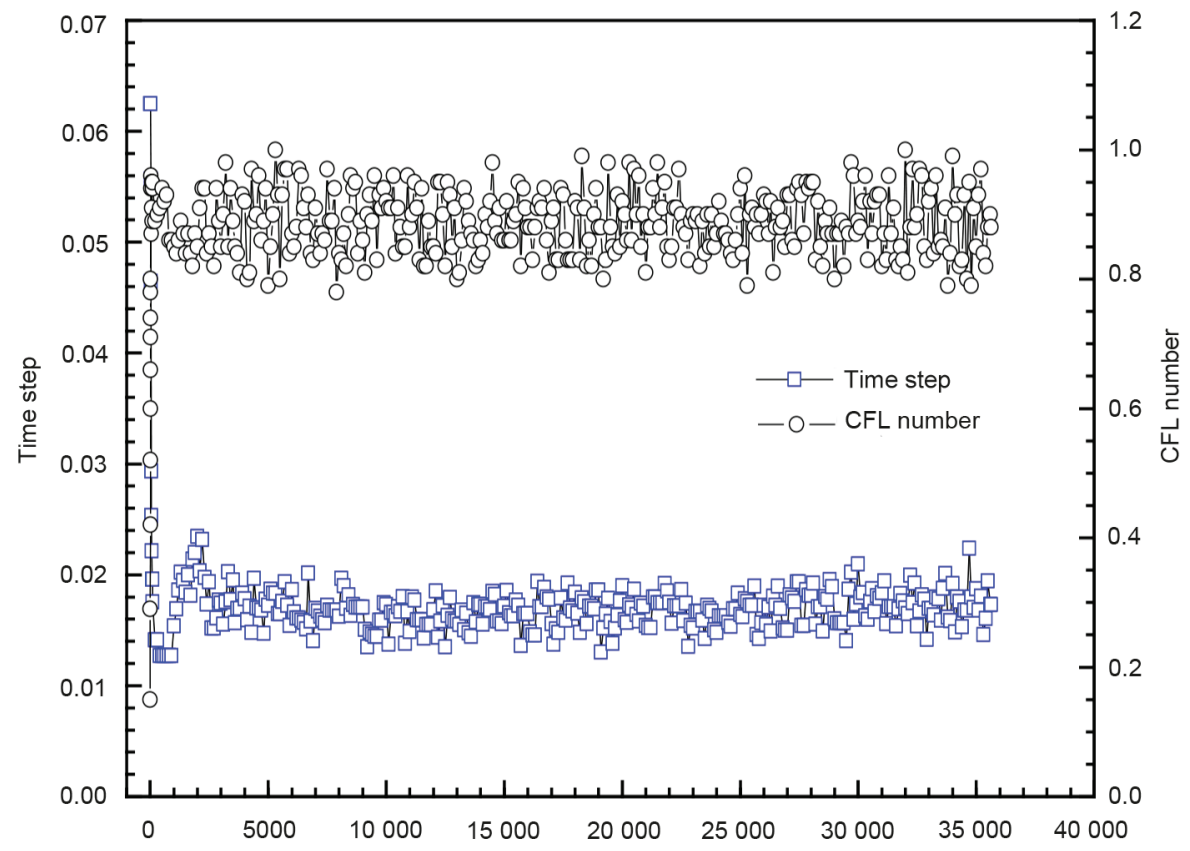

Fig. 3. Typical time step and CFL convergence of the simulation 


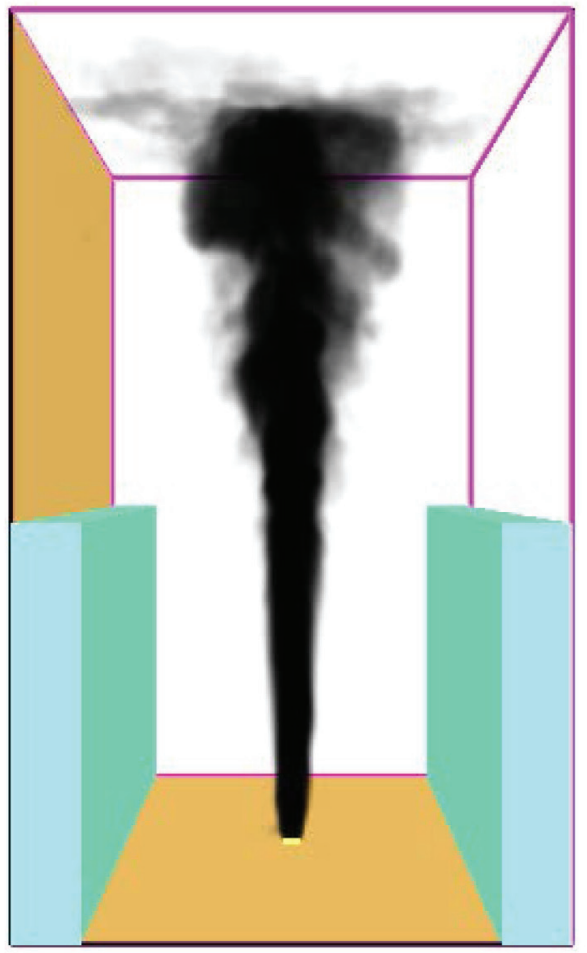

$u=0 \mathrm{~m} / \mathrm{s}$

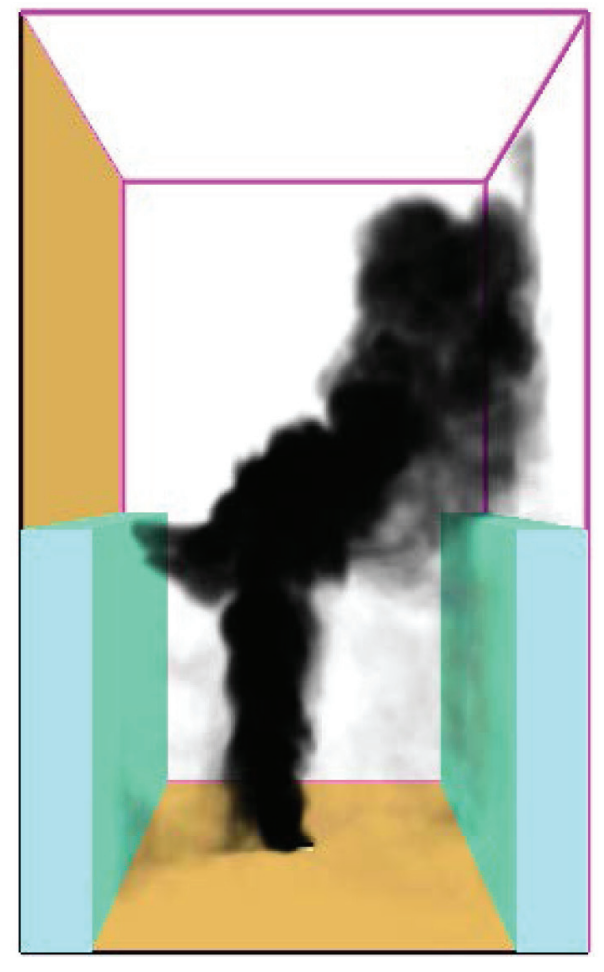

$u=3.0 \mathrm{~m} / \mathrm{s}$

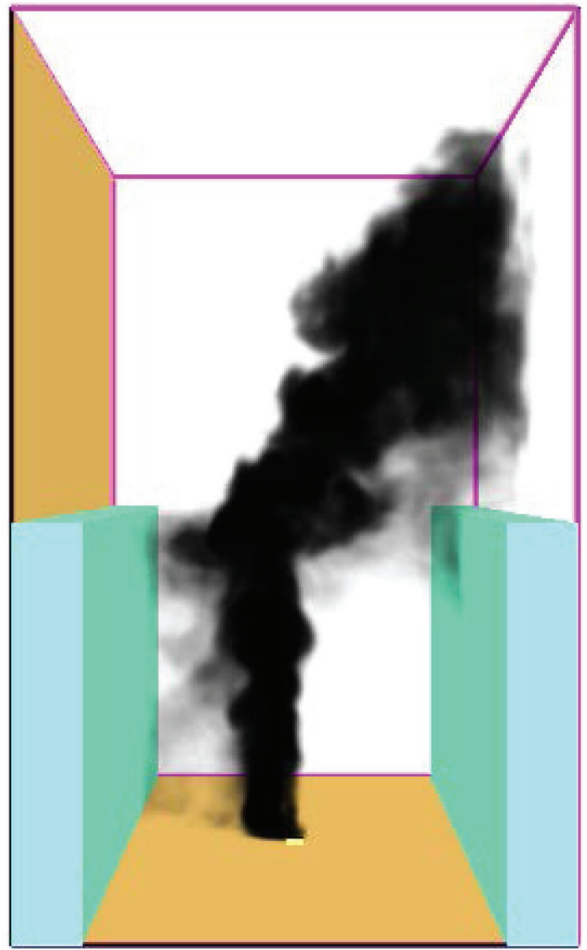

$u=2.8 \mathrm{~m} / \mathrm{s}$

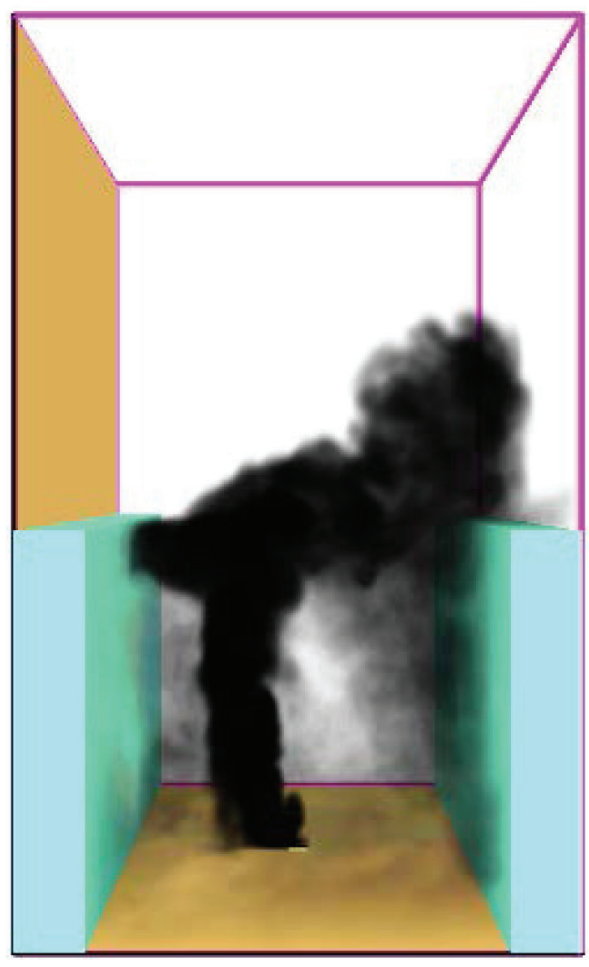

$u=5.0 \mathrm{~m} / \mathrm{s}$

Fig. 4. Smoke soot field and flow pattern under different wind velocities $(\mathrm{W}=\mathrm{H}=18 \mathrm{~m})$ 


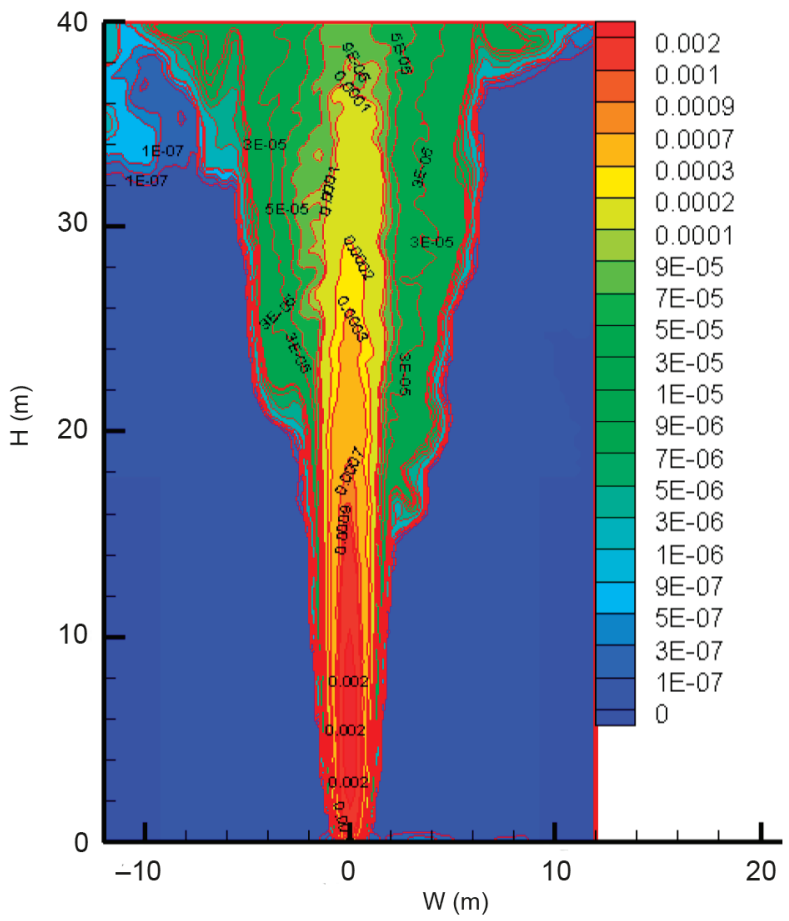

$u=0 \mathrm{~m} / \mathrm{s}$

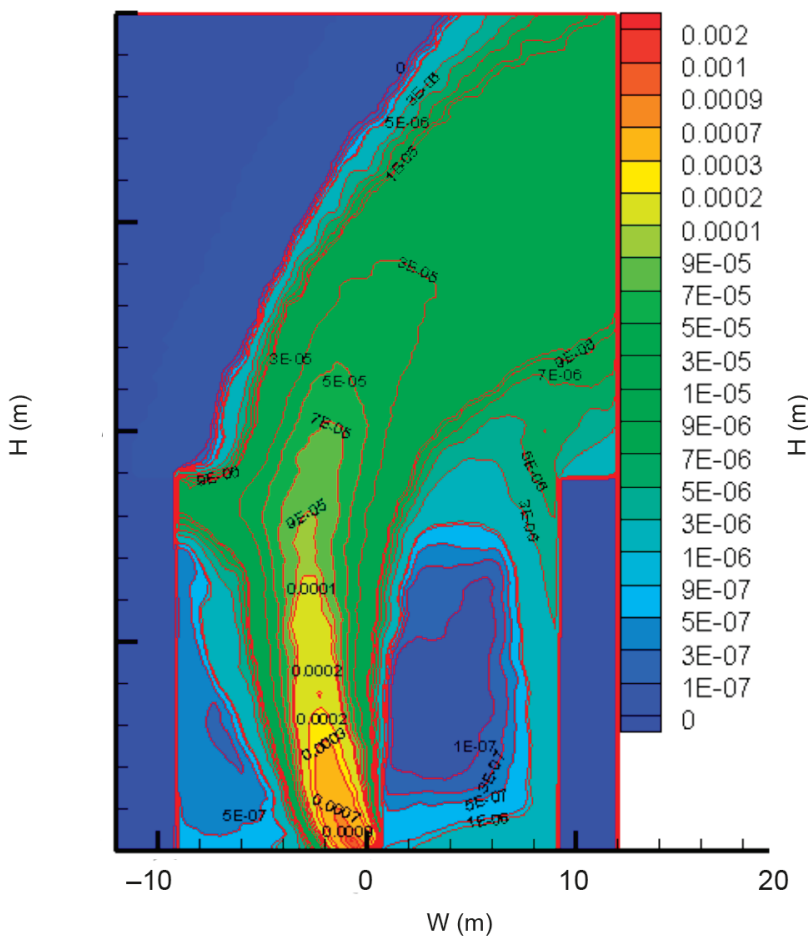

$u=3.0 \mathrm{~m} / \mathrm{s}$

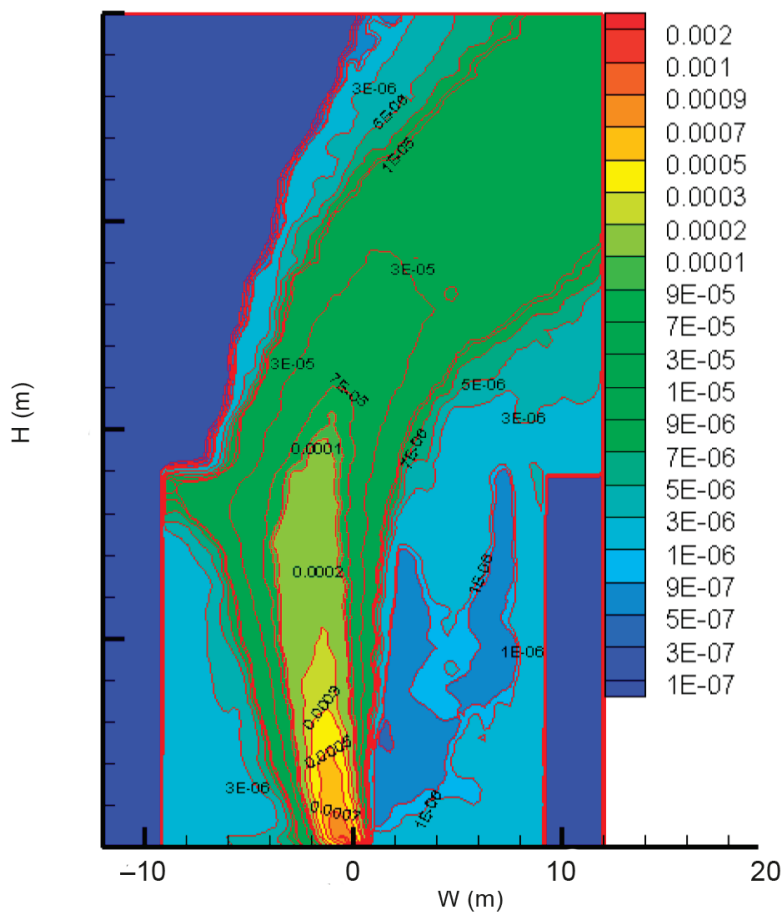

$u=2.8 \mathrm{~m} / \mathrm{s}$

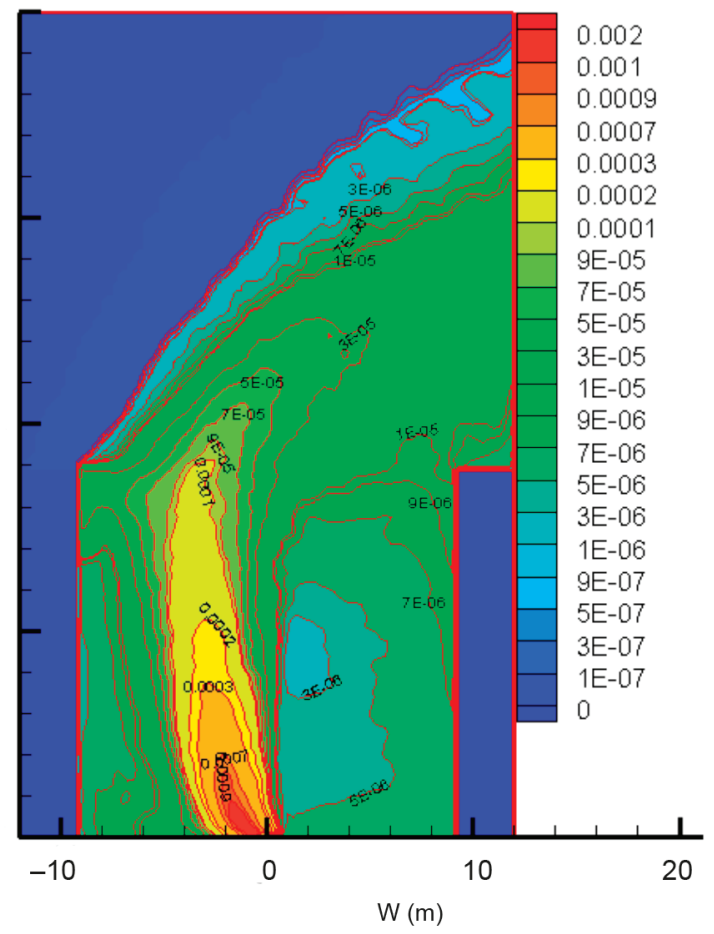

$u=5.0 \mathrm{~m} / \mathrm{s}$

Fig. 5. CO concentration contours under different wind velocities for the same HRR $(\mathrm{W}=\mathrm{H}=18 \mathrm{~m})$ 
plume begins to touch the leeward side wall at the top height of the canyon when the wind velocity reaches $2.8 \mathrm{~m} / \mathrm{s}$. When the wind velocity increased to be $3.0 \mathrm{~m} / \mathrm{s}$, the smoke begin to go downstream along the leeward side wall until it arrived at the bottom of the street canyon and starting going horizontally along the floor to the windward building. Thus, the re-circulation phenomenon takes place. The critical re-circulation wind velocity is then defined as such wind condition under which the smoke can go downstream along the leeward side wall and arrive at the bottom of the street canyon to then go horizontally along the floor to the windward building, as to form the re-circulation flow structure.

The critical re-circulation phenomenon is really a serious situation because the visibility in the atmosphere would be rather low, putting the vehicles and pedestrians in danger. If the wind velocity reached high levels, for example, $5.0 \mathrm{~m} / \mathrm{s}$ in Figures 4 and 5, the entire street canyon is full of dangerous fire smoke. Once the wind velocity exceeded the critical re-circulation velocity, the smoke is re-circulated back into street canyon and the vehicles and pedestrians would be in great danger. So, the critical re-circulation velocity should be studied as an important parameter.

\subsection{Critical re-circulation wind velocity}

Figure 6 presents the critical re-circulation wind velocities found for different street canyon heights. It can be observed that their relationship can be well correlated by a power law function with a power index of -0.32 , which is identical to the theoretical value of $-1 / 3$ indicated in Eqn (10). The critical Froude number values $\left(F r_{c}\right)$ are all shown to be near to 0.8 regardless of the height of the street canyon. This value is close to that found in our former work (0.7) (Hu et al. 2010) for the scenario of fire source at different floors in the windward adjacent building and ejecting smoke into the street canyon.

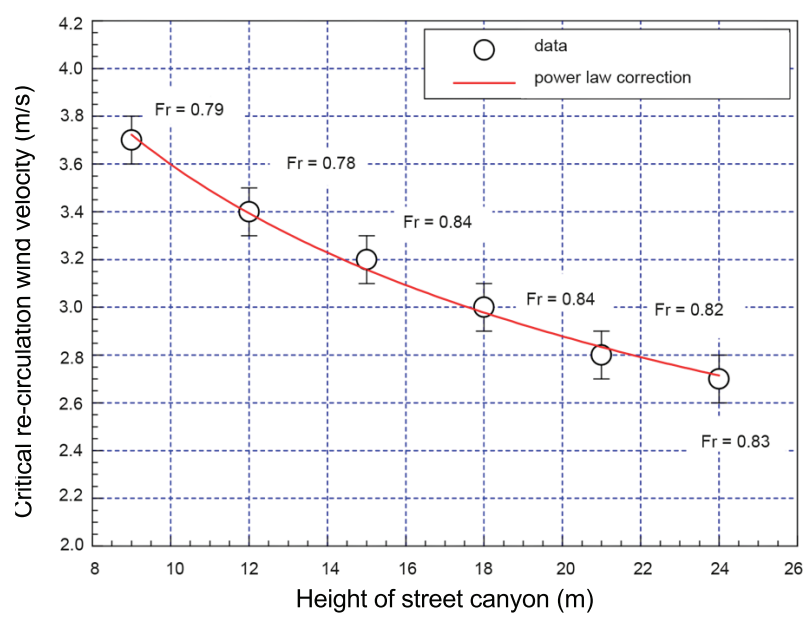

Fig. 6. Correlation of critical re-circulation wind velocity with street canyon height showing that their relation follows well with $-1 / 3$ power law function
Figure 7 collapses all the critical wind velocities found for different scenarios by the parameter of $\dot{Q}^{1 / 3} \cdot H^{-1 / 3}$. The scenarios include those reported in this paper, and in the earlier works for fire source in the windward adjacent building but at different floors $\left(H^{*}\right)$ in 2011 (Hu et al. 2010), as well as for a given height street canyon with different HRR ( $\dot{Q})$ (Hu et al. 2009). It is shown that all the data converge together and can be well collapsed by the proposed generalised parameter integrating the effects of both the buoyancy strength of the fire source and the characteristic height from the fire source to the top of the street canyon. The variation of the critical re-circulation wind velocity is in good proportional relationship with the integrated parameter of $\dot{Q}^{1 / 3} \cdot H^{-1 / 3}$. This is in good agreement with theoretically deduced relation of Eqn (10). However, it is noted that the regression line does not pass the origin of the axis. The intercept at the Y-axis is larger than zero. This is due to the fact that even the HRR $(\dot{Q})$ is very small (zero) or $H$ is very large, there still needs a certain level of wind velocity to form the large vortex flow inside the street canyon.

Figure 8 presents non-dimensional critical recirculation wind velocity $\frac{u_{c}}{\dot{Q}^{1 / 3} H^{-1 / 3}}\left(\frac{\rho c_{p} T_{\infty}}{g}\right)^{1 / 3}$ at different aspect ratios $(\mathrm{W} / \mathrm{H})$ in the skimming flow pattern $(\mathrm{W} / \mathrm{H}<1.43)$, which is widely focused in the former researches due to its special character of a large vortex formed inside the street canyon and its importance in pollutant dispersion. It can be observed that the variation trends in terms of the non-dimensional re-circulation wind velocity against aspect ratio fall into two distinct regimes with a turning point at the aspect ratio $(\mathrm{W} / \mathrm{H}=1)$. When the aspect ratio is less than 1, the non-dimensional critical re-circulation wind velocity is shown to increase linearly with aspect ratio. When the aspect ratio is larger than 1 , the critical re-circulation wind velocity is shown

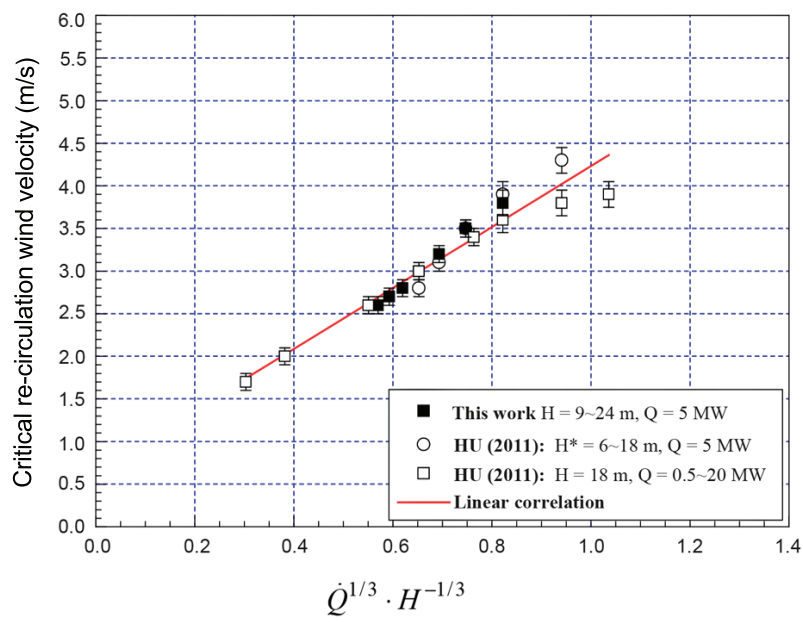

Fig. 7. Collapse and correlation of critical re-circulation wind velocity by the generalised integrated parameter $\dot{Q}^{1 / 3} \cdot H^{-1 / 3}$, which agrees well with the theoretical linear relation 


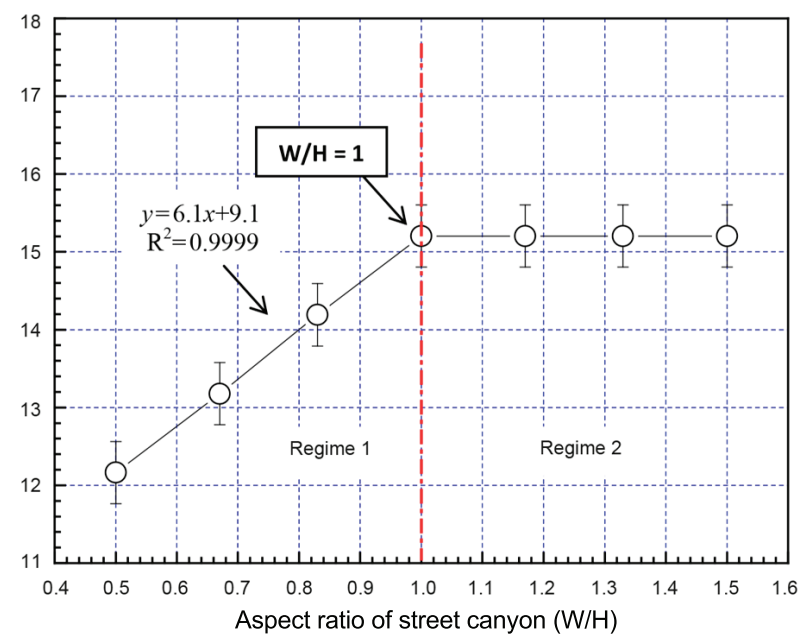

Fig. 8. Non-dimensional critical re-circulation wind velocity $\frac{u_{c}}{\dot{Q}^{1 / 3} H^{-1 / 3}}\left(\frac{\rho c_{p} T_{\infty}}{g}\right)^{1 / 3}$ against aspect ratio $(\mathrm{W} / \mathrm{H})$ of the street canyon in the skimming flow pattern $(\mathrm{W} / \mathrm{H}<1.43)$, showing two behavioural regimes with a turning point at $\mathrm{W} / \mathrm{H}=1$

to be maintained at constant. This yields following simply correlation function for the critical re-circulation wind velocity applicable in the skimming flow pattern:

For $\mathrm{W} / \mathrm{H}<1$

$$
\frac{u_{c}}{\dot{Q}^{1 / 3} H^{-1 / 3}}\left(\frac{\rho c_{p} T_{\infty}}{g}\right)^{1 / 3}=6.1\left(\frac{W}{H}\right)+9.1 .
$$

For $1<\mathrm{W} / \mathrm{H}<1.43$

$$
\frac{u_{c}}{\dot{Q}^{1 / 3} H^{-1 / 3}}\left(\frac{\rho c_{p} T_{\infty}}{g}\right)^{1 / 3}=15.2 .
$$

\section{Conclusions}

This paper reports the investigation on critical recirculation wind velocity for fire-induced pollutant smoke dispersion with strong buoyancy in urban street canyon by theoretical analysis and numerical simulation. A generalized universal relation applicable for any buoyancy strength and street canyon dimensions has been proposed. Major findings include:

1) The critical dimensionless Froude number $\left(F r_{c}\right)$, representing the balance of the wind flow effect and the buoyancy of the fire source, is found to be about $0.7 \sim 0.8$ in characterizing the corresponding critical re-circulation wind velocity.

2) The critical re-circulation wind velocity for fire source positioned at the ground base of a street canyon with different heights is proved to be in a relation of $-1 / 3$ power of the height of street canyon.

3) The critical re-circulation wind velocity is proportional to the generalised integrated parameter $\dot{Q}^{1 / 3} H^{-1 / 3}$. incorporating buoyancy strength $(\dot{Q})$ of the fire source and its height $(H)$ to the top of the street canyon. CFD simulation results agreed well with the theoretical relation for all different scenarios with various $\dot{Q}$ and $\mathrm{H}$, both for fire source positioned at the street ground base and at different floors of the windward adjacent building.

4) The effect of aspect ratio $(\mathrm{W} / \mathrm{H})$ on the critical recirculation wind velocity shows to fall into two behavioural regimes in the skimming flow pattern $(\mathrm{W} / \mathrm{H}<1.43)$. The non-dimensional critical recirculation wind velocity $\frac{u_{c}}{\dot{Q}^{1 / 3} H^{-1 / 3}}\left(\frac{\rho c_{p} T_{\infty}}{g}\right)^{1 / 3}$. firstly increases in the first regime with $\mathrm{W} / \mathrm{H}<1$, and then remains constant in the second regime with 1 $<\mathrm{W} / \mathrm{H}<1.43$, with a turning point at $\mathrm{W} / \mathrm{H}=1$. A global relation (Eqn (15)) is finally achieved and proposed for the critical re-circulation wind velocity $(u)$, fire heat release rate $(\dot{Q})$ and its height $(H)$ to the top of the street canyon as well as the street canyon aspect ratio $(\mathrm{W} / \mathrm{H})$.

\section{Acknowledgement}

This paper was supported by Nature Science Foundation of China under Grant No.40975005, National Basic Research Program of China under Grant No. 2012CB719702, Fundamental Research Funds for the Central Universities, and Program for New Century Excellent Talents in University under Grant No. NCET-09-0914.

\section{References}

Alarie, Y. 2002. Toxicity of fire smoke, CRC Critical Reviews in Toxicology 32(4): 259-289. http://dx.doi.org/10.1080/20024091064246

Baker, J.; Walker, H. L.; Cai, X. M. 2004. A study of the dispersion and transport of reactive pollutants in and above street canyons - a large eddy simulation, Atmospheric Environment 38(39): 6883-6892.

http://dx.doi.org/10.1016/j.atmosenv.2004.08.051

Besserre, R.; Delort, P. 1997. Recent studies prove that the main cause of death during urban fires is poisoning by smoke, Urgences Medicales 16(2): 77-80. http://dx.doi.org/10.1016/S0923-2524(97)88674-2

Chang, C. H. 2006. Computational fluid dynamics simulation of concentration distributions from a point source in the urban street canyons, Journal of Aerospace Engineering 19(2): 80-86.

http://dx.doi.org/10.1061/(ASCE)0893-1321(2006)19:2(80)

Chow, C. L.; Li, J. 2010. An analytical model on static smoke exhaust in Atria, Journal of Civil Engineering and Management 16(3): 372-381. http://dx.doi.org/10.3846/jcem.2010.42

Delichatsios, M. 2004. Tenability conditions and filling times for fires in large spaces, Fire Safety Journal 39(8): 643-662. http://dx.doi.org/10.1016/j.firesaf.2004.06.003

Hu, L. H.; Huo, R.; Yang, D. 2009. Large eddy simulation of fire-induced buoyancy driven plume dispersion in an urban street canyon under perpendicular wind flow, Journal of Hazardous Materials 166(1): 394-406. http://dx.doi.org/10.1016/j.jhazmat.2008.11.105

Hu, L. H.; Xu, Y.; Zhu, W.; Wu, L.; Tang, F.; Lu, K. H. 2010. Large eddy simulation of pollutant gas dispersion with buoyancy ejected from building into an urban street canyon, Journal of Hazardous Materials 192(3): 940-948. http://dx.doi.org/10.1016/j.jhazmat.2010.12.063 
Liu, H. Z.; Liang, B.; Zhu, F. Y.; Zhang, B. Y.; Sang, J. G. 2003. A laboratory model for the flow in urban street canyons induced by bottom heating, Advances in Atmospheric Sciences 20(4): 554-564. http://dx.doi.org/10.1007/BF02915498

Jonaitis, B.; Papinigis, V. 2005. Effect of long-term loading and fire temperatures on mechanical properties of concrete, Journal of Civil Engineering and Management 11(4): 283-288. http://dx.doi.org/10.1080/13923730.2005.9636359

Kim, J. J.; Baik, J. J. 2001. Urban street-canyon flows with bottom heating, Atmospheric Environment 35(20): 3395-3404.

http://dx.doi.org/10.1016/S1352-2310(01)00135-2

Kim, J. J.; Baik, J. J. 2010. Effects of street-bottom and building-roof heating on flow in three-dimensional street canyons, Advances in Atmospheric Sciences 27(3): 513-527. http://dx.doi.org/10.1007/s00376-009-9095-2

Konecki, M.; Półka, M. 2009. Simple fire model for comparative studies of critical conditions during combustion of chosen polymer materials, Journal of Civil Engineering and Management 15(3): 247-257. http://dx.doi.org/10.3846/1392-3730.2009.15.247-257

Lee, I. Y.; Park, H. M. 1994. Parameterization of the pollutant transport and dispersion in urban street canyons, Atmospheric Environment 28(14): 2343-2349. http://dx.doi.org/10.1016/1352-2310(94)90488-X

Liu, C. H.; Barth, M. C. 2002. Large-eddy simulation of flow and scalar transport in a modeled street canyon. Journal of Applied Meteorology 41(6): 660-673. http://dx.doi.org/10.1175/1520-0450(2002)041<0660:LESO $\mathrm{FA}>2.0 . \mathrm{CO} ; 2$

Liu, C. H.; Barth, M. C.; Leung, D. Y. C. 2004. Large-eddy simulation of flow and pollutant transport in street canyons of different building-height-to-street-width ratios, Journal of Applied Meteorology 43(10): 1410-1424. http://dx.doi.org/10.1175/JAM2143.1

Markatos, N. C.; Christolis, C.; Argyropoulos, C. 2009. Mathematical modeling of toxic pollutants dispersion from large tank fires and assessment of acute effects for fire fighters, International Journal of Heat and Mass Transfer 52(17-18): 4021-4030.

http://dx.doi.org/10.1016/j.ijheatmasstransfer.2009.03.039

McGrattan, K. B.; Hostikka, S.; Floyd, J. E.; Baum, H. R.; Rehm, R. G.; Mell, W. E. 2009. Fire dynamics simulator (version 5) technical reference guide, volume 1: mathematical model. NIST Special Publication, National Institute of Standards and Technology, US Department of Commerce, USA. 110 p.

McGrattan, K. B.; McDermott, R.; Hostikka, S.; Floyd, J. E. 2010. Fire dynamics simulator (version 5). User's guide. NIST Special Publication, National Institute of Stand- ards and Technology, US Department of Commerce, USA. $224 \mathrm{p}$.

Nakamura, Y.; Oke, T. R. 1988. Wind, temperature and stability conditions in an east-west oriented urban canyon, Atmospheric Environment (1967) 22(12): 2691-2700. http://dx.doi.org/10.1016/0004-6981(88)90437-4

Sini, J. F.; Anquetin, S.; Mestayer, P. G. 1996. Pollutant dispersion and thermal effects in urban street canyons, Atmospheric Environment 30(15): 2659-2677. http://dx.doi.org/10.1016/1352-2310(95)00321-5

Sun, X. Q.; Hu, L. H.; Chow, W. K.; Xu, Y.; Li, F. 2011. A theoretical model to predict plume rise in shaft generated by growing compartment fire, International Journal of Heat and Mass Transfer 54(4): 910-920. http://dx.doi.org/10.1016/j.ijheatmasstransfer.2010.10.012

Tang, F.; Hu, L. H.; Delichatsios, M.; Lu, K. H.; Zhu, W. 2011. Experimental study on flame height and temperature profile of buoyant window spill plume from an under-ventilated compartment fire, International Journal of Heat and Mass Transfer 55(1-3): 93-101.

http://dx.doi.org/10.1016/j.ijheatmasstransfer.2011.08.045

Tominaga, Y.; Mochida, A.; Yoshie, R.; Kataoka, H.; Nozu, T.; Yoshikawa, M.; Shirasawa, T. 2008. AIJ guidelines for practical applications of CFD to pedestrian wind environment around buildings, Journal of Wind Engineering and Industrial Aerodynamics 96(10): 1749-1761. http://dx.doi.org/10.1016/j.jweia.2008.02.058

Uehara, K.; Murakami, S.; Oikawa, S.; Wakamatsu, S. 2000. Wind tunnel experiments on how thermal stratification affects flow in and above urban street canyons, Atmospheric Environment 34(10): 1553-1562. http://dx.doi.org/10.1016/S1352-2310(99)00410-0

Vardoulakis, S.; Fisher, B. E. A.; Pericleous, K.; Gonzalez-Flesca, N. 2003. Modelling air quality in street canyons: a review, Atmospheric Environment 37(2): 155-182. http://dx.doi.org/10.1016/S1352-2310(02)00857-9

Walton, A.; Cheng, A. Y S. 2002. Large-eddy simulation of pollution dispersion in an urban street canyon - Part II: idealised canyon simulation, Atmospheric Environment 36(22): 3615-3627. http://dx.doi.org/10.1016/S1352-2310(02)00260-1

Walton, A.; Cheng, A. Y. S.; Yeung, W. C. 2002. Large-eddy simulation of pollution dispersion in an urban street canyon - Part I: comparison with field data, Atmospheric Environment 36(22): 3601-3613. http://dx.doi.org/10.1016/S1352-2310(02)00259-5

Xia, J.; Leung, D. Y. C. 2001. Numerical study on flow over buildings in street canyon, Journal of Environmental Engineering 127(4): 369-376.

http://dx.doi.org/10.1061/(ASCE)0733-9372(2001)127:4(369)

Longhua HU. Graduated with a $\mathrm{PhD}$ degree in 2006 from the State Key Laboratory of Fire Science, University of Science and Technology of China. Now he is an Associate Professor of State Key Laboratory of Fire Science, University of Science and Technology of China. His research fields include building fire dynamics including enclosure fire combustion behaviour, ventilation and smoke control.

Xiaochun ZHANG. PhD student of State Key Laboratory of Fire Science, University of Science and Technology of China. His research interests include urban fire smoke dispersion behaviour, and fire smoke ventilation in large complex buildings.

Wei ZHU. Graduated with a PhD degree in 2006 from the State Key Laboratory of Fire Science, University of Science and Technology of China. Now he is the Vice-director of Beijing Research Center of Urban System Engineering. His research fields include fire safety engineering and safety evaluation in urban systems.

Zhi NING. Graduated with a PhD degree in 2009 from the University of Southern California. Now he is an Assistant Professor in the School of Energy and Environment in City University of Hong Kong. His research focuses on aerosol physicochemical and toxicological characterization from their sources to microenvironments and personal exposure.

Fei TANG. PhD student of State Key Laboratory of Fire Science, University of Science and Technology of China. His research interests include fire dynamics and façade fire behaviour in urban buildings. 